Relations industrielles

Industrial Relations

\title{
La constitution de la société par actions en droit comparé, par F. de Sola Canizarès, Les Editions de l'Epargne, Paris, 1959, 173 pp.
}

\section{Gaston Cholette}

Volume 16, numéro 2, avril 1961

URI : https://id.erudit.org/iderudit/1021816ar

DOI : https://doi.org/10.7202/1021816ar

Aller au sommaire du numéro

Éditeur(s)

Département des relations industrielles de l’Université Laval

ISSN

0034-379X (imprimé)

1703-8138 (numérique)

Découvrir la revue

Citer ce compte rendu

Cholette, G. (1961). Compte rendu de [La constitution de la société par actions en droit comparé, par F. de Sola Canizarès, Les Editions de l'Epargne, Paris, 1959, 173 pp.] Relations industrielles / Industrial Relations, 16(2), 272-273.

https://doi.org/10.7202/1021816ar

Tous droits réservés (C Département des relations industrielles de l’Université Laval, 1961
Ce document est protégé par la loi sur le droit d'auteur. L'utilisation des services d'Érudit (y compris la reproduction) est assujettie à sa politique d'utilisation que vous pouvez consulter en ligne.

https://apropos.erudit.org/fr/usagers/politique-dutilisation/ 
ci étant consacrée spécialement au monde du travail en dehors des relations patronales-syndicales proprement dites.

L'auteur se propose d'apporter à la littérature traitant des problèmes des relations patronales-ouvrières une contribution originale en envisageant sa matière par le truchement de la sociologie. Il n'y a pas de doute que ce sont les économistes qui se sont intéressés le plus jusqu'à aujourd'hui aux relations industrielles. L'ouvrage de Dubin a le mérite, sinon d'innover tout à fait, du moins celui d'employer l'outil de la sociologie dans un domaine qui en a un si grand besoin.

L'auteur insiste tout spécialement sur la théorie de l'organisation, et sur le processus de la prise des décisions au sein des syndicats ouvriers et des compagnies. Ce processus est évidemment envisagé dans le cas de décisions qui ont trait aux relations entre les deux groupes.

La première partie de l'ouvrage traite de la direction des entreprises et des principaux facteurs qui sont à l'origine des décisions de l'employeur relatives à ses relations avec les groupements ouvriers.

Dans une deuxième partie, l'auteur parle des syndicats ouvriers et des groupements para-syndicaux, de leurs objectifs dans un contexte de rapports de force, enfin des fondements de leurs décisions.

La négociation collective, comme on pouvait s'y attendre, constitue le coeur, le centre de l'ouvrage. L'auteur y consacre beaucoup plus d'espace qu'aux autres questions. Il la considère d'abord en tant que manifestation d'un rapport de forces, puis dans son influence sur le fonctionnement des entreprises. Un autre chapitre porte sur les conflits industriels et les grèves.

Enfin, dans une quatrième partie, l'auteur étudie l'impact des relations patronales-ouvrières sur la société en général, notamment sur la sécurité sociale et le bien-être.

Tous ceux qui sont mêlés aux négociations collectives ou à d'autres activi- tés qui se rattachent aux relations industrielles auraient avantage à lire cet ouvrage.

\section{Gaston Cholette.}

\section{La constitution de la société par actions} en droit comparé, par F. de Sola Canizarès, Les Editions de l'Epargne, Paris, 1959, 173 pp.

Il s'agit du premier volume d'une nouvelle collection, sous le titre de - Publications du Centre français de droit comparé ». Cet ouvrage est en même temps le premier tome d'un grand traité consacré aux sociétés par actions et préparé selon la méthode du droit comparé.

L'auteur commence par analyser la notion actuelle de la société par actions en droit comparé. Il remarque qu'on ne peut pas formuler une définition universelle de la société par actions. Les traits qui semblent essentiels ne sont pas admis dans tous les pays; de plus, les mêmes principes n'ont pas le même sens dans tous les pays. Même le principe de base, c'est-à-dire la responsabilité limitée de l'actionnaire, n'est pas une règle absolue. L'auteur fait un rapide tour d'horizon et en profite pour dégager à la fois les traits communs et les différences dans les législations nationales.

Le deuxième est une sorte de nomenclature des sources légales et la bibliographie sur le sujet. L'auteur classe sa matière selon les pays de Common Law, de l'Europe continentale, de l'Amérique latine, de l'URSS et des républiques populaires, de l'Afrique, de l'Asie et de l'Océanie.

Il aborde ensuite successivement la nature juridique de l'acte constitutif, sa forme et son contenu. Il s'agit ici d'une des parties les plus importantes de la présente étude. L'auteur ne retient que les traits essentiels et ne porte des jugements de valeur qu'avec beaucoup de circonspection et de sobriété.

Les chapitres suivants sont consacrés aux actionnaires, au capital, à la souscription privée et publique, au contrôle et à la publicité de la constitution et à la sanction des irrégularités de la cons- 
titution. On trouve à la fin de la plupart des chapitres une synthèse comparative.

Le volume se termine par une magnifique conclusion sur la notion de personnalité juridique, reconnue à des titres divers et avec des conséquences plus ou moins grandes dans tous les Etats. L'auteur laisse de côté les théories et constructions purement doctrinales et se réfère seulement aux solutions découlant du droit positif des différents pays. On y trouve notamment un exposé de la conception anglaise et de la conception germanique, autour desquelles gravitent tous les systèmes.

\section{Gaston Cholette.}

The World of Work, Industrial Society and Human Relations, par Robert Dubin, Prentice Hall, Englewood Cliffs N.J., 1958, 448 pp., $\$ 8.00$.

Ce volume porte sur le monde du travail dans la société industrielle américaine. L'étude a pour objet les comportements des divers agents qui évoluent dans ce monde complexe et leurs relations. La question des relations patronales-syndicales est délibérément laissée de côté, car l'auteur a oru bon, à cause de son importance, de lui consacrer un autre ouvrage spéciel (Working Union-Management Relations, Englewood Cliffs, Prentice Hall, 1958). Ces deux ouvrages se complètent et peuvent etre lues séparément ou être considérés comme les deux tomes d'un même traité.

L'auteur projette sur les problèmes qu'il considère un éclairage original au moyen de deux disciplines appropriées: la sociologie et la psychologie. Il observe et analyse une quantité énorme de faits scientifiquement contrôlés. Il cherche à découvrir ce que les gens font lorsqu'ils travaillent et les raisons, les mobiles qui sont à l'origine de leurs actes.

Le volume est divisé en cinq parties principales: une introduction sur le travail dans la société moderne, une deuxième partie sur les structures du monde du travail, une troisième sur la population ouvrière, une quatrième sur Porganisation, la mise en oeuvre des forces du travail, enfin une cinquième partie sur la direction des institutions da monde du travail.

Ceux qui s'intéressent aux relations du travail auront profit à parcourir ce volume et à l'utiliser pour mieux comprendre et résoudre un grand nombre de problèmes qui se posent dans leur activité professionnelle.

\section{Gaston Chotette.}

Les préventeurs, par Pierre Caloni, Société d'éditions françaises et internationales, Paris, 1960, 437 pp.

Ce livre porte sur la lutte contre les accidents du travail. Son titre un peu surprenant désigne la personne dont l'occupation principale est la prévention.

Il s'agit de l'histoire d'une réalisation personnelle de l'auteur dans les professions du bâtiment et des travaux publics. L'auteur raconte la tâche qu'il a entreprise à son retour de la guerre de 1914-1918 et qu'il a poursuivie jusqu'à aujourd'hui. C'est donc par le biais d'une expérience personnelle de l'auteur que le lecteur pénètre dans le monde de la prévention des accidents du travail dans le bâtiment et les travaux publics, à travers le dédale des obstacles administratifs et des changements de politique.

Ce qui intéressera tout spécialement ceux qui s'occupent de la prévention des accidents du travail dans la province de Québec et au Canada en général, c'est la description de la structure et des rouages des institutions qui ont été édifiées en France, où une collaboration organique entre ouvriers, syndicats, employeurs et fonctionnaires publics donne d'excellents résultats.

L'auteur consacre une partie de son ouvrage à des réflexions sur la nature humaine et ses réactions devant le risque, et sur la meilleure manière de comprendre la tâche de préventeur.

On trouve enfin, dans la dernière partie, une imposante série dannexes qui fournissent une abondante documentation. 